\title{
Etude du statut phylogénétique du bovin Kouri du lac Tchad à l'aide de marqueurs moléculaires
}

\author{
P. Souvenir Zafindrajaona ${ }^{1}$ V. Zeuh ${ }^{1}$ \\ K. Moazami-Goudarzi ${ }^{2}$ D. Laloë ${ }^{3}$ \\ D. Bourzat ${ }^{1}$ A. Idriss ${ }^{1}$ F. Grosclaude ${ }^{2}$
}

\begin{abstract}
Mots-clés
Bovin Kouri - Bos taurus - Bos indicus Polymorphisme biochimique - Microsatellite - Phylogénie - Afrique occidentale - Lac Tchad.
\end{abstract}

\begin{abstract}
Résumé
Le polymorphisme de 25 systèmes de marqueurs génétiques autosomaux (11 systèmes de groupes sanguins, 5 locus de lactoprotéines et 9 microsatellites) a été analysé dans un échantillon de la population de bovins Kouri du lac Tchad provenant de la zone en bordure du lac et d'îles localisées dans la préfecture de Bol. Les résultats obtenus, ainsi que les données déjà acquises sur différentes populations de taurins et de zébus africains et sur les races françaises, ont été traités par analyse factorielle des correspondances et par des méthodes de classification pour tenter de préciser le statut phylogénétique du Kouri. Selon cette étude, basée sur des marqueurs autosomaux, le Kouri se rapproche plus des zébus que des taurins africains alors que, dépourvu de bosse et possédant le chromosome $Y$ submétacentrique de Bos taurus, il est classé normalement parmi les taurins. II reste à évaluer la validité de diverses hypothèses explicatives, d'ailleurs non exclusives, différant par l'ancienneté postulée de l'apport de gènes de zébus : parenté du Kouri avec le Sanga du Sud de l'Afrique, introgression du zébu lors de son expansion sur le continent à partir du VIII siècle après J.-C., ou métissages, à l'époque moderne, avec les zébus entourant le bassin du lac Tchad.
\end{abstract}

\section{INTRODUCTION}

Le bovin Kouri, ou Boudouma, est un animal sans bosse, donc un taurin, élevé sur les îles et le littoral du lac Tchad, aux confins de quatre pays, le Cameroun, le Niger, le Nigeria et le Tchad. Il s'agit d'un animal de grande taille, présentant une ossature développée et dont le poids va de 400 à $500 \mathrm{~kg}$ chez les femelles et de 400 à $700 \mathrm{~kg}$ chez les mâles. Il se distingue surtout par son cornage spectaculaire, caractérisé par des cornes de grandes dimensions qui peuvent prendre des formes diverses, notamment bulbeuse ou en

1. Laboratoire de recherches vétérinaires et zootechniques de Farcha, BP 433, N'Djaména, Tchad

2. Laboratoire de génétique biochimique et de cytogénétique, Institut national de la recherche agronomique, 78352 Jouy-en-Josas Cedex, France

3. Station de génétique quantitative et appliquée, Institut national de la recherche agronomique, 78352 Jouy-en-Josas Cedex, France

Correspondance : goudarzi@biotec.jouy.inra.fr lyre haute, et dont la circonférence à la base peut atteindre 80 à $100 \mathrm{~cm}$. Sa robe est généralement blanche, la présence de taches noires ou rouges étant interprétée, à tort ou à raison, comme un indice de croisement avec des zébus. Ses aptitudes sont intéressantes, tant pour la production de viande que pour celle de lait (4 à 6 litres par jour environ). Les Kouris réputés «purs » sont plutôt localisés dans les zones insulaires où les éleveurs, appartenant surtout à l'ethnie Boudouma, les déplacent d'une île à l'autre en fonction des ressources fourragères. Ils exploitent la végétation aquatique (zones basses, bordures et îles flottantes) à base de graminacées et de cypéracées. A la périphérie du lac, le Kouri est souvent métissé avec des zébus Choa ou M'Bororo, conduits vers les rives par les éleveurs Foulbés et Peuls $(1,26)$.

Selon Epstein (5), les premiers bovins domestiques implantés en Afrique ont été des taurins à cornes longues provenant du foyer de domestication de l'Asie du Sud-Ouest. Introduits en Egypte dans la seconde moitié du cinquième millénaire avant J.-C., ils se sont ensuite répandus dans le tiers Est et Nord du continent. Au deuxième millénaire avant J.-C., une seconde vague, constituée de 
taurins à cornes courtes, s'est répandue en Afrique à partir du même foyer d'origine, en se substituant souvent au premier peuplement. Des zébus à bosse cervico-thoracique et thoracique ont été introduits graduellement par l'Egypte et par la côte orientale de l'Afrique à partir du deuxième millénaire avant J.-C. C'est en Afrique de l'Est, et notamment en Ethiopie, que leur introgression dans les populations préexistantes de taurins à longues cornes a conduit au type Sanga, bétail métis à longues cornes qui s'est ensuite répandu vers le sud du continent. La véritable expansion du zébu en Afrique, et plus précisément du zébu à bosse thoracique, s'est faite beaucoup plus tard, avec l'invasion arabe, à partir de 700 ans environ après J.-C. Selon Epstein (5), les zébus auraient été sélectionnés en Asie du Sud-Ouest à partir de la même base populationnelle que les taurins. Toutefois, Loftus et coll. (15) ont conclu, d'une analyse comparée de l'ADN mitochondrial, que le zébu indien (Bos indicus) aurait été domestiqué, peut-être dans la région de l'actuel Pakistan, à partir d'une population sauvage (Bos primigenius) différente de la population d'origine des taurins et ayant divergé de cette dernière depuis au moins 200000 ans. Selon la même équipe, toutes les populations de zébus africains résulteraient de l'introgression de ce zébu indien par la voie mâle dans les populations taurines préexistantes $(2,15,16)$.

Le Kouri est considéré comme étant, avec le N'Dama, une des deux races reliques du peuplement initial de l'Afrique par les taurins domestiques à cornes longues. Il est possible qu'il soit le lointain descendant des taurins à cornes gigantesques qui ont peuplé l'ensemble du Sahara pendant le «néolithique humide » (de 4500 à 2500 avant J.-C.) et qui sont représentés par de célèbres gravures et peintures rupestres. Au cours des dernières décennies, le métissage assez fréquent du Kouri avec des zébus à la périphérie du lac a fait craindre l'extinction de ce type original (26). Toutefois, les pratiques des éleveurs de la zone insulaire du lac Tchad, ainsi sans doute que les difficultés d'accès à cette zone, ont permis de préserver jusqu'ici une population d'animaux généralement considérés comme purs et dont les effectifs, estimés (sans qu'ils aient jamais été connus avec certitude) à environ 30 à 50000 (1), seraient en diminution.

L'objet du présent travail a été d'évaluer, à l'aide de diverses classes de marqueurs du génome (groupes sanguins, protéines du sang et du lait, microsatellites), les distances génétiques entre la population Kouri et diverses races ou populations de taurins et de zébus.

\section{MATERIEL ET METHODES}

\section{Echantillons}

Les prélèvements d'échantillons individuels de sang et de lait d'animaux Kouri ont été effectués d'octobre 1994 à février 1996 dans des troupeaux privés localisés dans la préfecture de Bol, en bordure et sur des îles du lac Tchad. La collecte a porté au total sur 169 échantillons de lait, 140 prises de sang destinées au typage des microsatellites après extraction de l'ADN et 94 prises de sang pour l'analyse des groupes sanguins, les trois types de prélèvements ayant souvent été effectués sur des animaux différents (tableau I). Des échantillons de sang destinés au typage des microsatellites ont également été prélevés sur 37 zébus M’Bororo et 33 zébus Choa dans des troupeaux en bordure du lac.

Les échantillons de sang destinés au typage des microsatellites et les échantillons de lait ont été congelés à $-20{ }^{\circ} \mathrm{C}$ jusqu'à leur expédition vers la France (Laboratoire de génétique biochimique et de cytogénétique). Les échantillons de sang destinés à l'analyse des groupes sanguins ont été expédiés par avion de Bol vers la France via N'Djaména au fur et à mesure de leur collecte. Ils ont été reçus dans un délai de 48 heures après leur prélèvement au Laboratoire d'analyses génétiques pour les espèces animales, Jouy-en-Josas.

Les échantillons et données concernant les autres populations étaient ceux de Grosclaude (8), Grosclaude et coll. (9) et Moazami-Goudarzi et coll. (20) pour les races françaises, Grosclaude et coll. (11), Queval et coll. (24) et Mahé et coll. (17) pour les bovins africains. Parmi les données de Queval et coll. (24), l'échantillon de Guinée a été retenu pour représenter le N'Dama, les échantillons du Burkina Faso et de Côte d'Ivoire, descendant tous deux d'acquisitions en pays Lobi, ont été regroupés pour représenter le Baoulé. Les échantillons de sang destinés au typage des microsatellites provenaient du ranch d'Etat de Samandeni, au Burkina Faso, dont le troupeau a été constitué à partir d'acquisitions au ranch de Marahoué, en Côte d'Ivoire, et, pour le Baoulé, du ranch d'Etat de Gaoua, au Burkina Faso. Par ailleurs, le zébu malgache a été ajouté à l'étude comme témoin externe de type Bos indicus, les données étant celles de Souvenir Zafindrajaona et Lauvergne (28). Les effectifs des animaux utilisés sont rappelés dans le tableau I.

\section{Systèmes génétiques polymorphes}

L'ensemble des systèmes génétiques polymorphes retenus dans ce travail est présenté dans les tableaux II et III.

\section{Groupes sanguins}

Les onze systèmes de groupes sanguins érythrocytaires (A, B, C, F, J, L, M, S, Z, R' et T') connus chez les bovins ont été analysés par isohémolyse (10). Comme dans les travaux de Grosclaude et coll. (9) sur les races françaises et ceux de Queval et coll. (24) sur les populations africaines, les systèmes complexes $\mathrm{B}, \mathrm{C}$ et $\mathrm{S}$, vraisemblablement codés par plusieurs gènes étroitement liés, ont été simplifiés et ramenés à ce qui peut être assimilé à des séries alléliques. Toutefois, au système $\mathrm{B}$, les particularités du zébu malgache (fréquence élevée de phénogroupes comportant à la fois $\mathrm{P}_{2}$ et $\mathrm{T}$ ) ont conduit à ajouter à la série hepta allélique antérieure $\left(\mathrm{G}_{1}, \mathrm{I}_{1}, \mathrm{~K}, \mathrm{P}_{2}, \mathrm{~T}, \mathrm{~J}\right.$ " et - $)$ un huitième allèle, $\mathrm{P}_{2} \mathrm{~T}$. Pour le reste, comme dans Grosclaude et coll. (9) et Queval et coll. (24), les phénogroupes possédant $\mathrm{I}_{1} \mathrm{~K}$ et $\mathrm{G}_{1} \mathrm{~K}$ ont été classés avec $\mathrm{K}$, $\mathrm{I}_{1} \mathrm{~T}$ avec $\mathrm{T}$ et $\mathrm{I}_{1} \mathrm{G}_{1}$ avec $\mathrm{G}_{1}$. Au système $\mathrm{C}$, la série hexa allélique retenue a été celle de Queval et coll. (24), soit $\mathrm{C}_{1}, \mathrm{C}_{2}, \mathrm{C}_{1} \mathrm{C}_{2}{ }_{2}, \mathrm{C}_{0}$ et (-), les deux derniers allèles étant spécifiques des populations africaines. Au système $S$, les résultats obtenus sur les zébus malgaches avec le réactif anti-U" ne présentant pas, pour des raisons inconnues, la fiabilité voulue, le facteur U" n'a été pris en compte dans aucune des populations, ce qui, par rapport à la série allélique précédemment retenue (24), a conduit à des regroupements. En revanche, trois allèles supplémentaires, présents dans certaines populations, ont été adjoints $\left(\mathrm{UH}^{\prime}, \mathrm{U}_{0}^{\prime}\right.$ et $\left.\mathrm{U}_{0} \mathrm{H}^{\prime \prime} \mathrm{H}^{\prime}\right)$. La série allélique retenue comptait ainsi, au total, 13 allèles.

\section{Lactoprotéines}

Le polymorphisme des caséines $\alpha_{\mathrm{s} 1}, \beta$ et $\kappa$, de l' $\alpha$-lactalbumine $(\alpha$-La) et de la $\beta$-lactoglobuline ( $\beta$-Lg) pris en compte dans le présent travail a fait l'objet d'une publication spécifique (17).

\section{Microsatellites}

Les 9 microsatellites (ETH 152, ETH 225, INRA , INRA 005, INRA 032, INRA 035, INRA 063, INRA 072, INRA 016) utilisés dans cette étude ont été choisis parmi les 17 déjà mis en œuvre pour l'étude des races françaises $(20,21)$. Le choix a été effectué en fonction, d'une part, de leurs caractéristiques techniques (bonne aptitude à l'amplification et interprétation aisée des typages) et, d'autre part, de leurs caractéristiques génétiques (nombre d'allèles, localisation et répartition dans le génome). L'extraction d'ADN 
Tableau I

Effectifs des échantillons de populations retenus pour les différentes classes de marqueurs (groupes sanguins, protéines du lait, microsatellites)

\begin{tabular}{|c|c|c|c|c|}
\hline \multirow[b]{2}{*}{ Pays } & \multirow[b]{2}{*}{ Populations } & \multicolumn{3}{|c|}{ Nombre d'animaux } \\
\hline & & $\begin{array}{l}\text { Groupes } \\
\text { sanguins }\end{array}$ & $\begin{array}{l}\text { Protéines } \\
\text { du lait }\end{array}$ & Microsatellites \\
\hline Tchad & $\begin{array}{l}\text { Kouri } \\
\text { Zébu M'Bororo } \\
\text { Zébu Choa }\end{array}$ & 94 & 169 & $\begin{array}{r}140 \\
37 \\
33\end{array}$ \\
\hline $\begin{array}{l}\text { Guinée } \\
\text { et Burkina Faso }\end{array}$ & N'Dama & 192 & 76 & 31 \\
\hline $\begin{array}{l}\text { Côte d'Ivoire } \\
\text { et Burkina Faso }\end{array}$ & Baoulé & 470 & 96 & 37 \\
\hline Burkina Faso & Zébu Peul soudanais & 138 & 1022 & \\
\hline Madagascar & Zébu malgache & 210 & 586 & 60 \\
\hline France & $\begin{array}{l}\text { Aubrac } \\
\text { Brune des Alpes } \\
\text { Flamande } \\
\text { Salers } \\
\text { Tarentaise } \\
\text { Bretonne Pie-N oire } \\
\text { Charolaise } \\
\text { Limousine } \\
\text { Montbéliarde } \\
\text { Normande } \\
\text { Holstein } \\
\text { Jersey } \\
\text { Maine-Anjou } \\
\text { Parthenaise } \\
\text { Vosgienne }\end{array}$ & $\begin{array}{r}156 \\
350 \\
400 \\
226 \\
271 \\
91 \\
435 \\
377 \\
396 \\
454\end{array}$ & $\begin{array}{r}94 \\
151 \\
298 \\
94 \\
286 \\
78 \\
154 \\
40 \\
350 \\
318\end{array}$ & $\begin{array}{l}40 \\
50 \\
47 \\
54 \\
50 \\
50 \\
50 \\
31 \\
50 \\
50\end{array}$ \\
\hline
\end{tabular}

génomique a été effectuée selon le protocole de Jeanpierre (13). Les modalités de détermination des génotypes par électrophorèse en gel de polyacrylamide-urée après PCR en présence d'amorces fluorescentes ont été décrites par Moazami-Goudarzi et coll. (20). Le nombre d'allèles détectés par population est donné dans le tableau III.

\section{Traitement des données}

\section{Calcul des fréquences alléliques}

Pour les systèmes génétiques à allèles codominants, les fréquences alléliques ont été établies par comptage direct (les données peuvent être disponibles sur demande). Pour les locus bialléliques comportant un allèle négatif, les fréquences alléliques ont été calculées par la méthode de la racine carrée, et pour les systèmes complexes, par la méthode d'itération de Ceppellini et coll. (3).

\section{Calcul des distances génétiques}

L'indice de « distance standard » de Nei (22) a été utilisé pour quantifier la ressemblance entre races.

\section{Analyse factorielle des correspondances}

Cette analyse, qui permet une représentation simultanée des observations et des variables (14), donc ici des races et des allèles, a été effectuée par la procédure CORRESP du progiciel RSAS (version 6, SAS Institute 1989).

\section{Construction des phénogrammes}

La représentation arborée de neighbour-joining (27) a été utilisée pour construire les phénogrammes à partir de la matrice des distances calculées selon Nei (22). L'analyse de la robustesse des arbres a été réalisée par le test de bootstrap $(4,6)$. Après 500 rééchantillonnages des marqueurs, un arbre de consensus majoritaire permettant de visualiser les concordances a été construit (18). Les calculs ont été effectués à l'aide du logiciel Phylip (7).

\section{RESU LTATS}

Comme l'indique le tableau I, les trois classes de marqueurs génomiques (groupes sanguins, protéines du lait, microsatellites) n'ont pas été analysées dans toutes les populations. Cet état de fait a conduit à considérer deux sous-ensembles de données :

- le polymorphisme des groupes sanguins et des protéines du lait, soit un total de 16 systèmes, dans 15 races ou populations, dont 5 africaines (Kouri, N’Dama, Baoulé, zébu malgache, zébu Peul soudanais) et 10 races françaises (tableau I) ;

- le polymorphisme des 9 microsatellites dans 16 races ou populations, dont 6 africaines (Kouri, N’Dama, Baoulé, zébu malgache, zébu Choa, zébu M'Bororo) et 10 races françaises, dont 5 communes avec le premier sous-ensemble (tableau I). 


\section{Tableau II}

Allèles pris en compte pour les systèmes des groupes sanguins et des lactoprotéines. Pour $A$ et $A Z^{\prime}$, etc., lire $A^{A}$, $A^{A Z^{\prime}} ;$; (-) désigne un allèle « négatif ». Les notations $C_{1 \text {. }}$ et $U^{\prime}{ }_{1}$. utilisées dans $Q$ ueval et coll. (24) ont été remplacées ici par $C_{0}$ et $U^{\prime}{ }_{0}$. Le nombre total d'allèles s'élève à 64 soit 48 allèles indépendants

\begin{tabular}{|c|c|c|c|c|c|c|c|c|c|c|c|c|c|c|c|}
\hline \multicolumn{16}{|c|}{ Systèmes génétiques } \\
\hline \multicolumn{11}{|c|}{ G roupes sanguins } & \multicolumn{5}{|c|}{ Lactoprotéines } \\
\hline A & B & C & $\mathbf{F}$ & $J$ & $\mathbf{L}$ & M & $\mathbf{S}$ & $\mathbf{Z}$ & $\mathbf{R}^{\prime}$ & $\mathbf{T}^{\prime}$ & $\alpha_{\mathbf{S 1}}-\mathrm{Cn}$ & $\beta-C n$ & $\kappa-C n$ & $\alpha$-La & $\beta-\mathbf{L g}$ \\
\hline$A$ & $\mathrm{G}_{1}$ & $\mathrm{C}_{1}$ & $\mathrm{~F}$ & $J$ & $\mathrm{~L}$ & $M_{1}$ & $\mathrm{H}^{\prime}$ & Z & $\mathrm{R}^{\prime}$ & $\mathrm{T}^{\prime}$ & B & $A^{1}$ & $A$ & $A$ & $A$ \\
\hline$A Z^{\prime}$ & $\mathrm{I}_{1}$ & $\mathrm{C}_{2}$ & V & $(-)$ & $(-)$ & $M^{\prime}$ & $\mathrm{UH}^{\prime}$ & $(-)$ & $(-)$ & $(-)$ & C & $A^{2}$ & B & $B$ & B \\
\hline \multirow[t]{11}{*}{$(-)$} & $\mathrm{K}$ & $\mathrm{C}_{1}$ & $(-)$ & & & $(-)$ & $U^{\prime}$ & & & & $\mathrm{D}$ & $A^{3}$ & J & & $D$ \\
\hline & $P_{2}$ & $\mathrm{C}_{2}$ & & & & & SS"H' & & & & $\mathrm{H}$ & B & & & \\
\hline & $\mathrm{P}_{2} \mathrm{~T}$ & $\mathrm{C}_{0}$ & & & & & $\mathrm{SH}^{\prime}$ & & & & & C & & & \\
\hline & $\mathrm{T}$ & $(-)$ & & & & & $U^{\prime}{ }_{2}$ & & & & & $\mathrm{D}$ & & & \\
\hline & J" & & & & & & UH"H' & & & & & & & & \\
\hline & $(-)$ & & & & & & $\mathrm{SH}^{\prime \prime}$ & & & & & & & & \\
\hline & & & & & & & $\mathrm{H}^{\prime \prime} \mathrm{H}^{\prime}$ & & & & & & & & \\
\hline & & & & & & & US"H' & & & & & & & & \\
\hline & & & & & & & $U_{0}^{\prime}$ & & & & & & & & \\
\hline & & & & & & & $\mathrm{U}_{0}{ }_{0} \mathrm{H}^{\prime \prime} \mathrm{H}^{\prime}$ & & & & & & & & \\
\hline & & & & & & & $(-)$ & & & & & & & & \\
\hline 3 & 8 & 6 & 3 & 2 & 2 & 3 & 13 & 2 & 2 & 2 & 4 & 6 & 3 & 2 & 3 \\
\hline
\end{tabular}

Tableau III

N ombre d'allèles des marqueurs microsatellites. Le nombre total d'allèles différents s'élève à 112 , soit 103 allèles indépendants

Nombre d'allèles par microsatellite

Populations

ETH 152 ETH 225 INRA $\kappa$ INRA 016 INRA 032 INRA 035 INRA 005 INRA 063 INRA 072

$\begin{array}{lccccccccc}\text { Kouri } & 6 & 14 & 7 & 9 & 11 & 9 & 6 & 5 & 12 \\ \text { N'D ama } & 4 & 8 & 5 & 7 & 3 & 3 & 2 & 5 & 6 \\ \text { Baoulé } & 3 & 5 & 2 & 7 & 7 & 3 & 3 & 3 & 5 \\ \text { Zébu Choa } & 6 & 7 & 4 & 8 & 9 & 7 & 8 & 5 & 10 \\ \text { Zébu M'Bororo } & 6 & 6 & 4 & 8 & 8 & 5 & 6 & 5 & 8 \\ \text { Zébu malgache } & 9 & 7 & 7 & 6 & 13 & 4 & 4 & 6 & 7 \\ \text { Races françaises } & 9 & 6 & 6 & 10 & 6 & 10 & 3 & 7 & 11 \\ \text { Nombre total d'allèles différents } & 12 & 16 & 10 & 11 & 16 & 13 & 10 & 9 & 16\end{array}$

Chacun de ces deux sous-ensembles a fait l'objet d'une analyse factorielle des correspondances (AFC) et d'une démarche de classification aboutissant à la construction d'un phénogramme.

\section{Analyse factorielle des correspondances}

Dans le cas du polymorphisme des groupes sanguins et des protéines du lait, l'AFC sépare trois groupes de populations : les races françaises, très agrégées, les races ouest africaines, plus dispersées, et le zébu malgache qui se situe assez nettement à l'écart. Au sein du groupe ouest africain, N'Dama et Baoulé sont très proches (figure 1). Au total, les deux premiers axes rendent compte de 57 p. 100 de la variance totale. Sur le premier axe, le zébu malgache, et à un moindre degré le zébu Peul soudanais et le Kouri, se détachent des autres populations. Les allèles contribuant le plus à la construction de cet axe sont $\mathrm{M}^{-}$(30,7 p. 100 de l'inertie de cet axe), $\alpha_{\mathrm{S} 1}-\mathrm{Cn}^{\mathrm{C}}\left(10,8\right.$ p. 100), $\mathrm{T}^{\mathrm{T}}$ (8,3 p. 100), $\mathrm{S}^{\mathrm{U}_{0}^{\prime}}(7,8$ p. 100) et $\mathrm{B}^{\mathrm{P}_{2} \mathrm{~T}}$ (7,6 p. 100). On rappellera que l'allèle $\mathrm{B}^{\mathrm{P}_{2} \mathrm{~T}}$ est, tout au moins pour les populations considérées, spécifique du zébu malgache. Par ailleurs, $\mathrm{S}^{\mathrm{U}^{\prime}}$, fréquent chez le zébu malgache $(0,2)$ existe aussi chez le N'Dama, le Baoulé et le zébu Peul soudanais, 


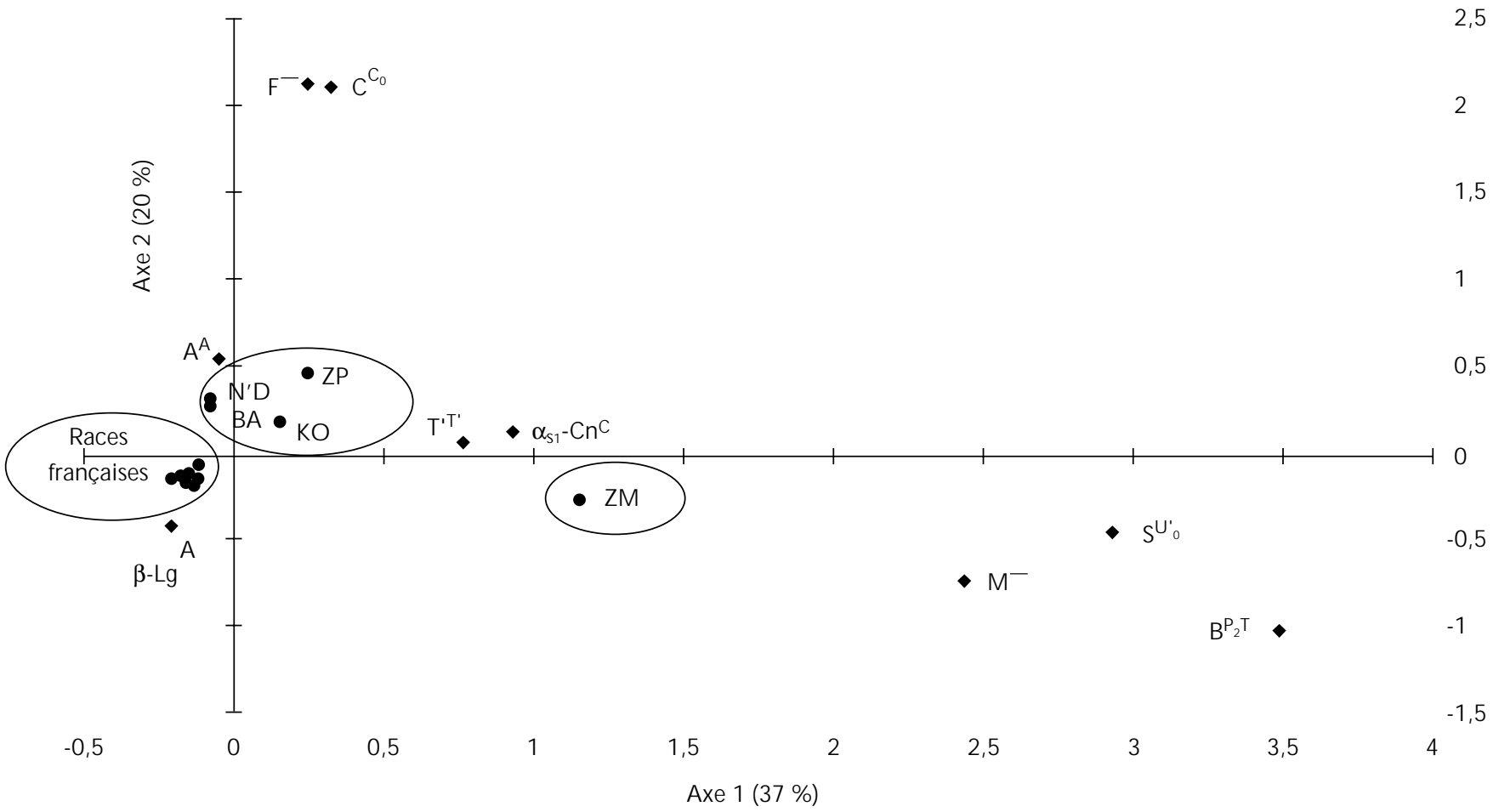

Figure 1 : analyse factorielle des correspondances appliquée aux données relatives aux groupes sanguins et aux lactoprotéines projection, sur le plan des deux premiers axes, des races bovines africaines et françaises et des neuf allèles les plus discriminants. BA : Baoulé ; N'D : N'Dama ; KO : Kouri ; ZM : zébu malgache ; ZP : zébu Peul soudanais ; AU : Aubrac ; BU : Brune des Alpes ; BR : Bretonne Pie-Noire ; CH : Charolaise ; FL : Flamande ; LI : Limousine ; MO : M ontbéliarde ; NO : Normande ; $\mathrm{SA}$ : Salers, TA : Tarentaise.

alors qu'il est absent des races françaises. L'effet des autres allèles discriminants est dû à des différences de fréquence, la plus connue étant la prédominance générale de l'allèle $\alpha_{\mathrm{S} 1}{ }^{-} \mathrm{Cn}^{\mathrm{C}}$ chez les zébus, qui s'oppose à celle de l'allèle $\alpha_{\mathrm{S} 1}-\mathrm{Cn}^{\mathrm{B}}$ chez les taurins $(17,19)$. Le second axe se caractérise surtout par la séparation des populations ouest africaines des autres populations. Les allèles contribuant le plus à la construction de cet axe sont $\mathrm{C}^{\mathrm{C}_{0}}\left(21,3 \mathrm{p}\right.$. 100) et $\mathrm{F}^{-}$ (7,7 p. 100) qui sont spécifiques des populations africaines (24), ainsi que $\beta-\mathrm{Lg}^{\mathrm{A}}\left(6,6\right.$ p. 100) et $\mathrm{A}^{\mathrm{A}}(5,6$ p. 100).

Dans le cas du polymorphisme des microsatellites, l'AFC identifie clairement quatre sous-ensembles : les races françaises, les taurins africains (Baoulé et N'Dama), les bovins du Tchad (zébu Choa, zébu M'Bororo et Kouri) et le zébu malgache (figure 2). Les deux premiers axes rendent compte de 54 p. 100 de la variance totale, valeur très proche de celle obtenue avec les groupes sanguins et les lactoprotéines. Le premier axe sépare les trois premiers groupes de races. Trois allèles contribuent principalement à sa construction, les allèles [191 pb] du microsatellite ETH 152, [174 pb] du microsatellite INRA $\kappa$ et $[157 \mathrm{pb}]$ du microsatellite ETH 225, qui expliquent respectivement 8,8 p. 100, 7 p. 100 et 7 p. 100 de l'inertie de l'axe. Le deuxième axe met surtout en évidence la distance entre les zébus malgaches et les autres populations. Les deux allèles qui influencent le plus cet axe sont l'allèle [213 pb] du microsatellite ETH 152, apparemment spécifique des zébus malgaches, et l'allèle [137 pb] du microsatellite ETH 225, qui contribuent respectivement à 33 et 10,3 p. 100 de l'inertie de l'axe.

\section{Phénogrammes}

La figure 3 représente le phénogramme obtenu à partir du polymorphisme des microsatellites. La séparation entre races françaises et populations africaines, caractérisée par une valeur maximale, est parfaitement tranchée. La proximité génétique des zébus tchadiens, Choa et M'Bororo, est également très nette, tout comme celle du Kouri avec ces zébus. Les deux types de taurins africains, N'Dama et Baoulé, sont également proches. Le phénogramme obtenu à partir des données sur les groupes sanguins et les lactoprotéines (non montré) donne des résultats comparables, mais avec des valeurs de bootstrap moins significatives, sauf pour la proximité du N'Dama et du Baoulé (93 p. 100).

\section{DISCUSSION}

Queval et Petit (25) ont publié, il y a 26 ans, dans cette même revue, un article donnant les fréquences alléliques des systèmes de groupes sanguins simples de la race Kouri (systèmes A, F, J, L, M, $\mathrm{Z}, \mathrm{R}^{\prime}, \mathrm{T}^{\prime}$ ) et les fréquences phénotypiques des facteurs antigéniques des systèmes complexes $(\mathrm{B}, \mathrm{C}, \mathrm{S})$. La seule différence vraiment significative entre les valeurs obtenues par ces auteurs et celles qui ont été estimées dans le présent travail concerne la fréquence de l'allèle $\mathrm{T}^{\mathrm{T}^{\prime}}(0,14$ pour Queval et Petit contre 0,64 dans cette étude). Il est vraisemblable que cette discordance n'est due qu'à une évolution de la spécificité du réactif anti-T', qui a été difficile à mettre au point. Pour les autres systèmes, les fréquences sont voisines, voire quasi identiques (identité à 0,01 près pour les systèmes $\mathrm{L}, \mathrm{Z}$ et $\mathrm{R}$ ', et à 0,02 près pour les facteurs antigéniques $\mathrm{I}_{1}, \mathrm{P}_{2}, \mathrm{~T}, \mathrm{~K}$ et J" du système B). Sur la base de ces observations, il est permis de considérer que la population Kouri n'a guère évolué au cours du dernier quart de siècle.

Le présent travail avait pour objectif de préciser la position génétique du Kouri parmi les populations de taurins et de zébus de l'Afrique de l'Ouest. Le fait que le polymorphisme des marqueurs « classiques », groupes sanguins et lactoprotéines, et celui des 


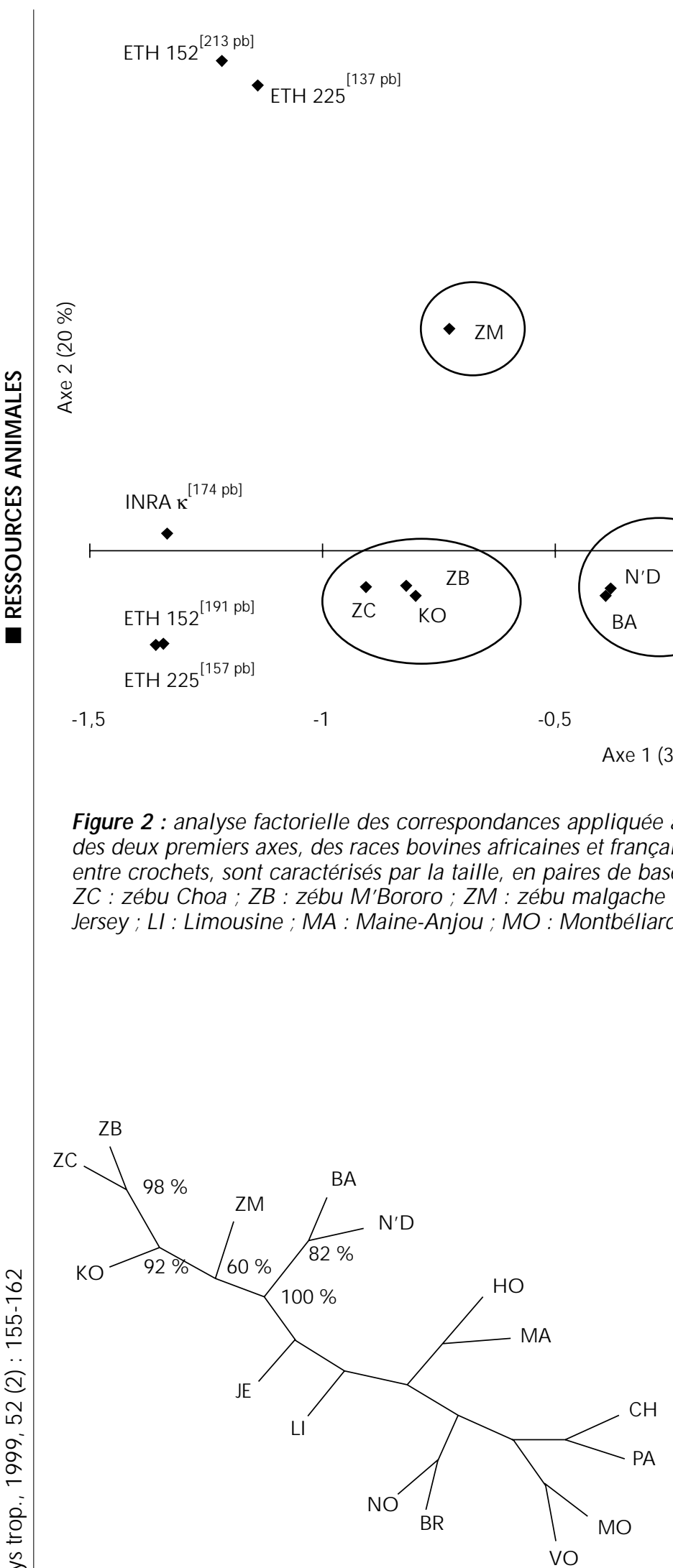

Figure 3 : arbre de consensus obtenu après 500 ré-échantillonnages des données (microsatellites). Les valeurs de bootstrap inférieures à 50 p. 100 ne sont pas indiquées. BA : Baoulé ; N'D : N'D ama ; KO : Kouri ; ZC : zébu Choa ; ZB : zébu M'Bororo ; ZM : zébu malgache ; BR : Bretonne Pie-Noire ; $\mathrm{CH}$ : Charolaise ; HO : Holstein ; JE : Jersey ; LI : Limousine ; MA : Maine-Anjou ; MO : Montbéliarde ; NO : Normande; PA : Parthenaise; VO : Vosgienne. microsatellites n'aient pu être analysés dans toutes les populations considérées a conduit à effectuer deux analyses séparées, basées, pour l'une, sur les 48 allèles indépendants des 16 marqueurs classiques et, pour l'autre, sur les 103 allèles indépendants des 9 microsatellites. Ce traitement fractionné présente toutefois l'intérêt de permettre la comparaison des résultats de deux analyses indépendantes et d'apprécier ainsi le degré de concordance de ces résultats.

Dans l'analyse factorielle des correspondances basée sur les microsatellites, le Kouri s'agrège sans ambiguité avec les zébus africains. En revanche, dans l'analyse basée sur les marqueurs classiques, il apparaît plus ou moins intermédiaire entre les taurins africains et le zébu Peul soudanais. Cette position intermédiaire du Kouri est particulièrement nette dans le cas des protéines du lait (17). Il faut toutefois souligner que, dans la présente analyse, les trois locus permettant selon Queval et coll. (24) la meilleure discrimination entre les taurins et les zébus, ceux de trois protéines sanguines (hémoglobine, transferrine, albumine), n'ont pas été pris en compte. Il est probable que l'adjonction de ces locus aurait conduit à séparer beaucoup plus nettement, comme dans Queval et coll. (24), les zébus africains des taurins. Or les fréquences chez le Kouri des allèles $\mathrm{Hb}^{\mathrm{B}}(0,381$ selon Petit et Queval (23), et 0,520 selon Souvenir Zafindrajaona et Zeuh (29)) et $\mathrm{Tf}^{\mathrm{F}}(0,296$ selon les derniers auteurs) rapprochent fortement le Kouri des zébus. La prise en compte des locus de protéines sanguines aurait donc, de toute évidence, rapproché le Kouri des zébus, et éloigné ce groupe de celui des taurins africains et européens. Il ressort ainsi que le Kouri, qui est un taurin puisqu'il n'a pas de bosse et possède le chromosome Y de Bos taurus, est plus proche, par ses marqueurs autosomaux, des zébus que des taurins. 
Ces résultats sont à rapprocher de ceux de MacHugh et coll. (16) qui, en se basant sur la fréquence relative des chromosomes Y spécifiques de Bos taurus et de Bos indicus, et sur celle d'allèles de microsatellites considérés comme provenant du zébu indien, ont conclu à l'existence d'un gradient d'introgression de ce zébu de l'est vers l'ouest de l'Afrique, phénomène qui aurait procédé de la seule voie mâle, et aurait même affecté le N'Dama, population taurine de l'extrême Ouest africain. On notera toutefois, à ce stade, qu'aucun de la douzaine de caryotypes de mâles Kouri examinés jusqu'ici n'était de type Bos indicus $(12,23)$. Chez ce taurin du lac Tchad, le phénomène postulé par MacHugh et coll. (16) ne serait donc décelable qu'au niveau du polymorphisme des marqueurs autosomaux.

Toutefois, la question des origines du Kouri paraît rester très ouverte. Il serait intéressant de savoir si la présence des allèles rapprochant ce taurin du zébu est la conséquence des métissages qui se sont produits en bordure du lac Tchad au cours des dernières décennies ou si, d'après la thèse de MacHugh et coll. (16), elle résulterait d'une introgression bien antérieure qui serait la conséquence de l'expansion du zébu vers l'ouest de l'Afrique, ellemême liée à l'invasion de ce continent par les Arabes. Mais on ne peut pas non plus exclure l'hypothèse que des particularités génétiques du Kouri aient une origine plus ancienne et que ce bovin soit apparenté aux animaux Sanga du Sud de l'Afrique ou même, à la limite, qu'il ait conservé, plus que le N'Dama, des allèles de la population domestique originelle de taurins à cornes longues de l'Asie du Sud-Ouest (5). Ces diverses possibilités ne s'excluent d'ailleurs pas les unes les autres.

Pour tenter de répondre à ces questions, il conviendrait en premier lieu de compléter les données de cette étude sur les systèmes marqueurs qui n'ont pas été examinés dans certaines populations (protéines sanguines et protéines du lait) pour permettre une étude unifiée de toutes les races ou populations considérées. Il serait aussi utile de procéder à l'examen d'un nombre plus élevé de caryotypes mâles, pour savoir si le chromosome Y de Bos indicus peut être détecté chez le Kouri réputé pur, même à faible fréquence. Par ailleurs, l'analyse dans cette population des 10 microsatellites ayant servi dans les travaux de MacHugh et coll. (16) à déceler le phénomène d'introgression du zébu indien permettrait de vérifier si le Kouri peut relever du phénomène décrit par ces auteurs. Deux seulement de ces 10 microsatellites sont inclus dans cette étude (ETH 152 et ETH 225). Enfin, des investigations sur des populations de type Sanga à l'aide de la même série de marqueurs moléculaires permettraient de confirmer ou d'infirmer l'hypothèse d'une parenté du Kouri avec le Sanga.

\section{Remerciements}

Cette étude a été réalisée dans le cadre du projet Conservation de la race bovine Kouri dans le bassin conventionnel du lac Tchad, financé par l'Union européenne, Commission des communautés européennes, Direction générale du développement (DG VIII/A/1, ligne budgétaire B7 - 5040/93/24). Nous tenons à exprimer notre plus vive gratitude aux personnalités dont le soutien constant a permis sa réalisation, notamment son altesse Mboudou M. Youssouf, Chef de Canton de Bol, M. A.Ch. Mahamat, alors Ministre de l'Elevage et de l'hydraulique de la République du Tchad et M. G. Durazzo, alors Conseiller pour le Développement rural à la Délégation de l'Union européenne en République du Tchad. Nous remercions la Sodelac (Société de développement du Lac) pour son accueil et son aide logistique, ainsi que les différents services administratifs, l'équipe du secteur vétérinaire de Bol, les autorités locales de la Préfecture du Lac ainsi que les éleveurs eux-mêmes qui, par leur accueil, ont beaucoup facilité notre tâche sur le terrain. Nous sommes particulièrement reconnaissants au Cirdes
(Centre international de recherche-développement sur l'élevage en zone subhumide) et notamment à Issa Sidibé pour la fourniture d'échantillons de sang indispensables pour mener cette étude à bonne fin. Ce travail a bénéficié de l'aide efficace, pour les analyses et les traitements de données, du département de génétique animale de l'Inra (Laboratoire de génétique biochimique et de cytogénétique) et du Gie Labogena (Laboratoire d'analyses génétiques pour les espèces animales).

\section{BIBLIO GRAPHIE}

1. BOURZAT D., SOUVENIR ZAFINDRAJAONA P., 1996. L'élevage du bœuf Kouri dans la zone lacustre du bassin du lac Tchad. In : Atlas d'élevage du bassin du lac Tchad. Wageningen, Pays-Bas, CTA, p. 88-90.

2. BRADLEY D.G., MACHUGH D.E., CUNNINGHAM P., LOFTUS R.T., 1996. Mitochondrial diversity and the origin of African and European cattle. Proc. natl. Acad. Sci. USA, 93: 5131-5135.

3. CEPPELLINI R., SINISCALCO M., SMITH C.A.B., 1956. The estimation of gene frequencies in a random mating population. Ann. Hum. Genet. 20: $97-115$.

4. EFRON B., 1979. Bootstrap methods: an other look at the jacknife. Ann. Stat., 7: 1-26.

5. EPSTEIN H., 1971. The origin of domestic animals of Africa, Vol. 1. New-York, NY, USA, Africana publishing, p. 208-212.

6. FELSENSTEIN J., 1985. Confidence limits on phylogenies: an approach using the bootstrap. Evolution, 39: 783-791.

7. FELSENSTEIN J., 1993. PHYLIP (Phylogeny inference package), Version 3.5. Seattle, WA, USA, Washington University, Department of Genetics, $275 \mathrm{p}$.

8. GROSCLAUDEF., 1988. Le polymorphisme génétique des principales lactoprotéines bovines. Inra Prod. Anim., 1 : 5-17.

9. GROSCLAUDE F., AUPETIT R.Y., LEFEBVRE J., MERIAUX J.C., 1990 Essai d'analyse des relations génétiques entre les races bovines françaises à l'aide du polymorphisme biochimique. Genet. Sel. Evol., 22 : 317-338.

10. GROSCLAUDE F., GUERIN G., HOULIER G., 1979. The genetic map of the $B$ system of cattle blood groups as observed in French breeds. Anim. Blood Groups biochem. Genet., 10: 199-218.

11. GROSCLAUDE F., MAHE M.F., MERCIER J.C., 1974. Comparaison du polymorphisme génétique des lactoprotéines du zébu et des bovins. Ann. Génét. Sél. Anim., 6 : 305-329.

12. HANOTTE O., OKOMO M., BRADLEY D., VERJEE Y., OCHIENG J. TEALE A., REGE J.E.O., 1998. Geographic distribution and frequency of taurine Bos taurus and zebu Bos indicus $Y$ chromosome haplotypes amongst sub-Saharan African cattle breeds (Poster). In: XXVI International Conference on Animal Genetic, Aukland, Australia, August 1998.

13. JEANPIERRE M., 1987. A rapid method for purification of DNA from blood. Nucleic Acids Res., 15: 9611

14. LEBART L., MORIBEAU A., PIRON M., 1995. Statistique exploratoire multidimensionnelle. Paris, France, Dunod, $439 \mathrm{p}$.

15. LOFTUS R.T., MACHUGH D.E., BRADLEY D.G., SHARP P.M., CUN N INGHAM P., 1994. Evidence for two independent domestications of cattle. Proc. natl. Acad. Sci. USA, 91: 2757-2761.

16. MACHUGH D.E., SHRIVER M.D., LOFTUSR.T., CUN NINGHAM P. BRADLEY D.G., 1997. Microsatellites DNA variation and the evolution, domestication and phylogeography of taurine and zebu cattle (Bos taurus and Bos indicus). Genetics, 146: 1071-1086.

17. MAHE M.F., MIRANDA G., QUEVAL R., BADO A., SOUVENIR ZAFINDRAJAONA P., GROSCLAUDE F., 1999. Genetic polymorphism of milk proteins in African Bos taurus and Bos indicus populations Characterization of variants $\alpha_{51}-\mathrm{Cn}^{H}$ and $\kappa-\mathrm{Cn}$. Genet. Sel. Evol. 31: 239-253.

18. MARGUSH T., MCMORRIS F.R., 1981. Consensus N-trees. Bull. Math. Biol., 43: 239-244.

19. MERCIER J.C., GROSCLAUDE F., 1993. Génétique moléculaire de protéines du lait et de leurs gènes. In : Martinet J., Houdebine L.M. éd. Biologie de la lactation. Paris, France, Inserm/Inra, p. 319-347. 
20. MOAZAM I-GOUDARZI K., LALOE D., FURET J.P., GROSCLAUDE F., 1997. Analysis of genetic relationships between 10 cattle breeds with 17 microsatellites. Animal Genet., 28: 338-345.

21. MOAZAMI-GOUDARZI K., VAIMAN D., MERCIER D., GROHS C., FURET J.P., LEVEZIEL H., MARTIN P., 1994. Emploi de microsatellites pour I'analyse de la diversité génétique des races bovines françaises : premiers résultats. Genet. Sel. Evol., 26 : 155-165.

22. NEI M., 1972. Genetic distance between populations. Am. Nat, 106: 283-292.

23. PETIT J.P., QUEVAL R., 1973 : Le Kouri : race bovine du lac Tchad. II. Etude biochimique : les hémoglobines et les constituants du sérum. Revue Elev. Méd. vét. Pays trop., 26 : 97-104.

24. QUEVAL R., MOAZAMI-GOUDARZI K., LALOE D., MERIAUX J.C., GROSCLAUDE F., 1998. Relations génétiques entre populations de taurins ou zébus d'Afrique de l'O uest et taurins européens. Genet. Sel. Evol., 30 : 367-383.

\section{Summary}

Souvenir Zafindrajaona P., Zeuh V., Moazami-G oudarzi K., Laloë D., Bourzat D., Idriss A., G rosclaude F. Study on the phylogenetic status of Lake Chad Kuri cattle using molecular markers

The polymorphism of 25 autosomal genetic marker systems (i.e., 11 blood group systems, 5 lactoproteins loci and 9 microsatellites) was analyzed in a sample of the Lake Chad Kuri cattle population originating from shore areas and islands located in the Bol district. The results obtained, together with those previously reported for various populations of African taurine and zebu cattle, and for French breeds, were submitted to correspondence analysis and classification methods in an attempt to specify the phylogenetic status of the Kuri. Since the Kuri is humpless and has Bos taurus submetacentric $Y$ chromosome, it is normally classified as taurine cattle. However, according to the present study based on autosomal markers the Kuri appears to be closer to zebu than to African taurine cattle. Non exclusive divergent explanations as to which period the zebu gene admixture can be traced back remain to be evaluated: relationship between Kuri and Sanga cattle of Southern Africa, introgression of the zebu during its expansion through the continent from the eighth century A.D. onwards or crossbreeding with zebus located around Lake Chad in recent times.

Key words: Kuri cattle - Bos taurus - Bos indicus Biochemical polymorphism - Microsatellite - Phylogeny W est-Africa - Lake Chad.
25. QUEVAL R., PETIT J.P., 1973. Le Kouri : race bovine du lac Tchad. III. Les facteurs érythrocytaires. Revue Elev. Méd. vét. Pays trop., 26 : 235-244.

26. QUEVAL R., PETIT J.P., TACHER G., PROVOST A., PAGOT J., 1971. Le Kouri, race bovine du lac Tchad. I. Introduction générale à son étude zootechnique et biochimique : origines et écologie de la race. Revue Elev. Méd. vét. Pays trop., 24 : 667-687.

27. SAITOU N., NEI M., 1987. The neighbour-joining method: a new method for reconstructing phylogenetic trees. Mol. biol. Evol., 4: 406-425.

28. SOUVENIR ZAFINDRAJAONA P., LAUVERGNE J.J., 1993. Comparaison de populations de zébu malgache à l'aide des distances génétiques. Genet. Sel. Evol., 25 : 749-771.

29. SOUVENIR ZAFIDRAJAONA P., ZEUH V., 1996. Volet de travaux génétiques. In : Projet de "Conservation de la race bovine Kouri dans le bassin conventionnel du lac Tchad », rapport final. N'D jaména, Tchad, Laboratoire de recherches vétérinaires et zootechniques de Farcha, p. 16-66.

Reçu le 1.12.98, accepté le 22.9.99

\section{Resumen}

Souvenir Zafindrajaona P., Zeuh V., Moazami-Goudarzi K., Laloë D., Bourzat D., Idriss A., Grosclaude F. Estudio del estadio filogenético del bovino Kuri del lago Chad, mediante marcadores moleculares

Se analizó el polimorfismo de 25 sistemas de marcadores genéticos autosómicos (11 sistemas de grupos sanguíneos, 5 locus de lactoproteínas y 9 microsatélites), en una muestra de la población de bovinos Kuri del lago Chad, provenientes de la zona periférica del lago y de las islas localizadas en el municipio de Bol. Los resultados obtenidos, así como los datos adquiridos sobre las diferentes poblaciones de taurinos y de cebúes africanos y sobre las razas francesas, fueron tratados mediante análisis factorial de correspondencia y por los métodos de clasificación, para intentar precisar el estadio filogenético del Kuri. Según el presente estudio, basado en marcadores autosómicos, el Kuri se acerca más de los cebúes que de los taurinos africanos, aunque, desprovisto de giba y portador del cromosoma $Y$ submetacéntrico del Bos taurus, se clasifica normalmente entre los taurus. Q ueda por determinar la validez de las diversas hipótesis explicativas, no exclusivas, diferiendo por la antigüedad postulada del aporte genético de los cebúes: parentesco del Kuri con el Sanga del Sur de Africa, introgresión del cebú durante la expansión de la raza en el continente a partir del siglo VIII D.C. o mestizajes durante nuestra época, con los cebúes que rodean la meseta del lago Chad.

Palabras clave : Ganado bovino Kuri - Bos taurus - Bos indicus - Polimorfismo bioquímico - Microsatélite - Filogenia Africa occidental - Lago Chad. 